\title{
Bringing patient centricity to diabetes medication access in Canada
}

Judith L Glennie'

Katharina Kovacs Burns ${ }^{2,3}$

Paul Oh $\mathrm{Oh}^{4,5}$

'J. L. Glennie Consulting Inc., Aurora, ON, ${ }^{2}$ School of Public Health,

University of Alberta, ${ }^{3}$ Alberta Health Services, Edmonton, $A B,{ }^{4}$ Cardiac

Rehabilitation and Secondary

Prevention Program, UHN, ${ }^{5}$ Toronto

Rehabilitation Institute and Peter

Munk Cardiac Centre, Toronto,

ON, Canada
Correspondence: Judith L Glennie J. L. Glennie Consulting Inc., II I Rush Road, Aurora, ON L4G 7G5, Canada

Tel +l 9057265846

Email Judith_glennie@on.aibn.com
This article was published in the following Dove Press journal:

ClinicoEconomics and Outcomes Research

17 October 2016

Number of times this article has been viewed

\begin{abstract}
Canada must become proactive in addressing type 2 diabetes. With the second highest rate of diabetes prevalence in the developed world, the number of Canadians living with diabetes will soon reach epidemic levels. Against international comparisons, Canada also performs poorly with respect to diabetes-related hospitalizations, mortality rates, and access to medications. Diabetes and its comorbidities pose a significant burden on people with diabetes (PWD) and their families, through out-of-pocket expenses for medications, devices, supplies, and the support needed to manage their illness. Rising direct and indirect costs of diabetes will become a drain on Canada's economy and undermine the financial stability of our health care system. Canada's approach to diabetes medication assessment and funding has created a patchwork of medication access across provinces. Access to treatments for those who rely on public programs is highly restricted compared to Canadians with private drug plans, as well in contrast with public payers in other countries. Each person living with diabetes has different needs, so a "patient-centric" approach ensures treatment focused on individual circumstances. Such tailoring is difficult to achieve, with the linear approach required by public payers. We may be undermining optimal care for PWD because of access policies that are not aligned with individualized approaches and increasing overall health care costs in the process. The scope of Canada's diabetes challenge demands holistic and proactive solutions. Canada needs to get out from "behind the eight ball" and get "ahead of the curve" when it comes to diabetes care. Improving access to medications is one of the tools for getting there. Canada's "call to action" for diabetes starts with effective implementation of existing best practices. A personalized approach to medication access, to meet individual needs and optimize outcomes, is also a key enabler. PWD and prescribers need reimbursement approaches that allow them to use existing tools (ie, medications and supplies) to manage diabetes in a timely manner and to avoid and/or delay major downstream complications. Keywords: diabetes, patient-centered care, reimbursement, policy, pharmaceuticals, health technology assessment
\end{abstract}

\section{Scope of the Canadian diabetes challenge}

Canada faces a significant challenge in managing the burden of type 2 diabetes mellitus (T2DM) on people with diabetes (PWD) and the health care system. Canada has the second highest rate of diabetes prevalence in the developed world and performs poorly against international comparators with respect to key clinical metrics, including diabetes-related hospitalizations, mortality rates, and access to medications. ${ }^{1}$

According to a 2015 report from the Canadian Diabetes Association (CDA), "Diabetes prevalence has more than doubled since 2000 (8.9\% of the population [ie, 3.34 million people] in 2015), and will grow by more than $40 \%$ by 2025 , along with a similar increase 
in direct health care costs to treat diabetes." ${ }^{2}$ It is estimated that diabetes and its related conditions cost the health care system CAD \$3 billion annually in direct costs. Juxtaposed to this significant societal and economic impact are the paradoxical barriers for PWD and providers in obtaining access to recommended care, education, support services, and the tools (ie, prescription medications and supplies) to manage their diabetes, thus impairing their ability to delay and/or prevent diabetes complications.

While the potential economic impact of diabetes on society has been well articulated, the growing burden of diabetes also has an impact on PWD. As part of their efforts to make sure that our societal response to diabetes stays focused on these individuals, the CDA published the Diabetes Charter for Canada (2014) to describe the rights and responsibilities of PWD and to drive improvements in programs and the delivery of health services for them. ${ }^{3}$ While it is envisioned that the principles and values promoted within the Charter will become the "new standard" for people living with diabetes, many of the statements currently reflect key areas of challenge in achieving personalized, high-quality diabetes care.

For instance, medication access is a key right and responsibility noted in several sections of the Charter, given the central role played by medications in diabetes management. One of the primary rights outlined for Canadians living with diabetes is "Affordable and timely access to prescribed medications, devices, supplies and high quality care...." In terms of government, the responsibilities cited include: "Guarantee fair access to diabetes care, education, prescribed medications, devices, and supplies to all Canadians, no matter what their income or where they live." Unfortunately, there has been little progress on this aspect of the Charter, and access to diabetes medications continues to be a challenge on several fronts.

The 2015 CDA report ${ }^{2}$ documented the high out-ofpocket costs for diabetes care and treatment, placing significant financial burden on low-income Canadians and those without adequate insurance coverage. According to the report, 15\% of PWD indicated a lack of government and/ or private prescription drug coverage, while another $30 \%$ of PWD reported a lack of insurance to cover blood glucose monitoring supplies or equipment.

Figures related to out-of-pocket costs are significant, with the report citing the following statistics:

- Type 1 diabetes: CAD\$1,074 and CAD\$4,909 a year, on average.

- T2DM: CAD\$723-CAD\$1,914 a year, on average.

- Seniors with T2DM: pay 36\%-70\% of diabetes treatment costs out of pocket.
The impact of these coverage gaps is significant, with $25 \%$ of PWD reporting that the cost of medications, supplies, and devices affected their ability to adhere to their diabetes treatment and, in some cases, it resulted in not filling their prescriptions at all due to the cost, thus compromising their ability to manage their diabetes.

\section{Canada's current approach to diabetes medication management: processes and impacts}

In Canada, each provincial and territorial government offers a drug benefit plan for eligible groups, as does the federal government for the eligible populations under its specific jurisdiction (ie, Correctional Service of Canada, First Nations and Inuit Health Branch, and Veterans Affairs Canada). Most provincial/territorial drug insurance systems involve public programs with supplemental private coverage that consider eligibility and deductibles based on income. Most jurisdictions have specific programs for population groups that may require more enhanced coverage for high medication expenses, including seniors, recipients of social assistance, and individuals with specific diseases or conditions that are associated with high drug costs. ${ }^{4}$

International comparisons reveal that overall medication access in Canada appears to be more restrictive than what PWD experience in other countries. A 2016 report from Innovative Medicines $\mathrm{Canada}^{5}$ examined the comparative reimbursement and availability of new medications in 20 of the top 30 Organization for Economic Cooperation and Development countries, chosen based on similarity to Canada in terms of social and economic factors and the availability of complete reimbursement data (countries examined included those in Europe, South East Asia, Australia, New Zealand, and the USA). When a representative "basket" of products was examined (ie, products that were reimbursable across provinces accounting for at least $80 \%$ of the eligible national public drug plan population), Canada ranked 18th of the 20 countries, with only $37 \%$ of new medicines receiving public reimbursement across jurisdictions. Furthermore, Canadian public drug plans placed reimbursement restrictions on $90 \%$ of new medicines, which made Canada rank 17th among the 20 countries. $^{5}$

\section{Processes}

To understand the basis for the current state of medication access in Canada, it is important to understand the rather lengthy and complex processes by which PWD ultimately get access to these new products. As in other countries, medicines in Canada 
are approved for sale through the National Regulatory Agency (Health Canada). Subsequently, to be considered for public formulary listing (in all provinces besides Quebec), new medicines undergo a national health technology assessment (HTA) through the Canadian Agency for Drugs and Technologies in Health (CADTH) - specifically, the Common Drug Review process. (Within Quebec, l'Institut national d'excellence en santé et services sociaux provides the analogous function. ${ }^{6}$ )

In formulating their drug reimbursement recommendations, CADTH and the l'Institut national d'excellence en santé et services sociaux analyze studies - from the manufacturer as well as from a broader literature search - that report on the clinical effectiveness, safety, and cost-effectiveness of the drugs under review. Drugs are compared with currently funded treatment(s) to determine the therapeutic advantages and disadvantages of the new drugs, as well as the comparative or incremental cost-effectiveness. ${ }^{7}$

Subsequent to the HTA review, a confidential public reimbursement price is negotiated by the pan-Canadian Pharmaceutical Alliance ${ }^{8}$ on behalf of all provinces. Provinces and federal drug plans (advised by their respective formulary evaluation committees) then independently make the final decision on reimbursement for their covered population, based on the HTA recommendation and any jurisdictionspecific reviews that may be needed, taking into consideration local practices, prior decisions, as well as political and economic issues. This may lead to different listing decisions in public plans across the country.

In addition to the regulatory, HTA, and reimbursement processes outlined above, jurisdictions frequently reevaluate the evidence available for a group of products to determine if changes are needed to their funding status and/or the listing criteria. In the case of diabetes agents, CADTH has conducted several reviews of individual and classes of medications over the last decade. A recently published network meta-analysis and pharmacoeconomic assessment acknowledged that while both older and newer therapies achieved improved glucose control, agents such as older biphasic insulin and sulfonylureas (SUs) were deemed to be more effective and cost-effective than newer oral and injectable agents on the basis of hemoglobin A1c (HbA1c) reduction and comparatively low acquisition price. ${ }^{9}$ Unfortunately, these reports have not thoroughly considered other efficacy and safety parameters, particularly those that are important to PWD (eg, nocturnal hypoglycemia, weight gain, etc). For instance, despite acknowledging the statistical significance of decreased weight loss in clinical trials and the availability of utility data related to weight changes from third-party sources, the analysis undertaken in the 2013 CADTH review ${ }^{9}$ did not assign a utility value to weight loss associated with glucagon-like peptide-1 (GLP-1) analogs in either the reference-case or sensitivity analyses. If such parameters were included in the assessment of diabetes medications, one would expect the cost-effectiveness to improve and, thus, increase the likelihood of positive reimbursement recommendations and potentially broader patient access.

\section{Impacts}

In general, the narrow focus of CADTH's evidence assessment process has resulted in recommendations that restrict the use of newer diabetes agents. Indeed, examination of publicly available recommendations through the Common Drug Review ${ }^{10}$ shows that several newer insulins and the GLP-1 agonists were not recommended for listing. In addition, newer oral agents such as dipeptidyl peptidase-4 inhibitors (DPP-4s) and sodium-glucose cotransporter-2 inhibitors (SGLT2s) were recommended for restricted use; that is, only as combination therapies when standard agents such as metformin, SUs, and neutral protamine Hagedorn (NPH) insulin were insufficient or intolerable. Furthermore, in its reviews of newer classes such as DPP-4s, the advisory committee seemed to be looking for impact well beyond HbAlc, such as effects on micro- and macrovascular outcomes, as well as an assessment of the relationship between $\mathrm{HbAlc}$ and cardiovascular $(\mathrm{CV})$ outcomes specifically associated with each new molecule. While such data are relevant over the life cycle of a drug, the requirement for this type of information at the time of market approval would unduly delay the availability of new medications for PWD by many years.

From a patient-centricity perspective, while CADTH's processes do invite formal input from patient organizations, it is unclear how this information is factored into the aforementioned expert committee recommendations. For instance, patient group commentary into the recent review of a GLP-1 agent ${ }^{11}$ pointed out that currently available therapies often have limitations and that the goal of access to new classes of medications is to achieve better diabetes control with minimal or no side effects (especially hypoglycemia), no weight gain, and improved life and life expectancy without diabetes complications. Furthermore, PWD expressed a clear desire to address the negative psychological and emotional impact associated with diabetes and its treatments. None of these are factors were seemingly taken into consideration in the CADTH review of this and other diabetes medications.

The restrictive nature of the national HTA review process has cascaded further in terms of access limitations for 
diabetes medications on public plans. This is best demonstrated by a recent overview of provincial listings compiled by the CDA, ${ }^{12}$ which summarized the reimbursement status of products across provinces as follows (as of April 4, 2016): glyburide and metformin - listed; DPP-4s - mostly restricted; GLP-1s - mostly not listed; long-acting basal analog insulins - mostly not listed or restricted; SGLT2s - canagliflozin restricted, while others are not yet listed; and thiazolidinediones - mostly restricted or not listed. In addition, there are differences in medication access for PWD depending on which province they reside (Table 1). As seen in the CDA compilation, access to many diabetes medications is restricted for Atlantic Canadians in comparison to Ontarians, despite the high prevalence of diabetes in the Atlantic provinces.

\section{What are clinicians saying they need?}

As articulated by the 2015 CDA report, there are many screening, diagnostic, and monitoring gaps between the care that PWD receive and the recommendations outlined in the CDA's 2013 evidence-based clinical practice guidelines. ${ }^{13}$

Regarding the medications for diabetes management, the 2013 guidelines provide a road map for optimal treatment of T2DM founded on several key principles:

Table I Examples of differences in diabetes medication access through government drug plans across Canada (as of April 2016)

\begin{tabular}{|c|c|c|}
\hline Medication & Funded & Not funded \\
\hline \multirow[t]{7}{*}{ Glimepiride } & Ontario & British Columbia \\
\hline & New Brunswick & Alberta \\
\hline & Prince Edward Island & Saskatchewan \\
\hline & Newfoundland & Nova Scotia \\
\hline & Manitoba (restricted) & FNIHB/Nunavut \\
\hline & Quebec (restricted) & Yukon \\
\hline & Northwest Territories (restricted) & \\
\hline \multirow[t]{11}{*}{ Saxagliptin } & Ontario & Newfoundland \\
\hline & Quebec & Yukon \\
\hline & British Columbia (restricted) & \\
\hline & Alberta (restricted) & \\
\hline & Saskatchewan (restricted) & \\
\hline & Manitoba (restricted) & \\
\hline & New Brunswick (restricted) & \\
\hline & Nova Scotia (restricted) & \\
\hline & Prince Edward Island (restricted) & \\
\hline & FNIHB/Nunavut (restricted) & \\
\hline & Northwest Territories (restricted) & \\
\hline Canagliflozin & $\begin{array}{l}\text { All other Canadian provinces and } \\
\text { territories (restricted) }\end{array}$ & British Columbia \\
\hline \multirow[t]{2}{*}{ Liraglutide } & Quebec (restricted) & All other Canadian \\
\hline & Northwest Territories (restricted) & $\begin{array}{l}\text { provinces and } \\
\text { territories }\end{array}$ \\
\hline
\end{tabular}

Abbreviation: FNIHB, First Nations and Inuit Health Branch.
- Do not wait too long to initiate pharmacotherapy.

- Match the right drug to the right patient.

- Increase doses in a timely fashion.

- Add medications in a timely fashion (ie, medication intensification).

The issue of tailoring choices among available medications to the needs of the PWD is emphasized in the guidelines, given the varying characteristics of each medication and the comorbidities that may predispose an individual PWD to adverse outcomes.

In reality, achieving effective diabetes control quickly with minimal side effects is a significant challenge for both PWD and prescribers. ${ }^{14}$ Studies have documented the magnitude of delays in the addition of oral diabetes medications (ie, medication intensification) despite suboptimal HbA1c levels. ${ }^{15}$ A UK retrospective cohort study of 81,573 people with T2DM demonstrated that the median time from treatment initiation to intensification exceeded the maximum follow-up time of the study (ie, 7.2 years). Such delays in intensification resulted in prolonged periods of hyperglycemia that, intuitively, undermine the objectives of high-quality diabetes care. Similar conclusions have been drawn in similar studies in other countries. ${ }^{16-19}$

If a PWD does not have insurance coverage and cannot afford the medication their physician believes is the best option, there is clearly a high likelihood of negative clinical impact. Even those PWD with private drug plans exhibit compromised medication adherence if they have high outof-pocket drug costs, again undermining optimal care. ${ }^{20}$ Individuals with government drug plan coverage who cannot access the medication deemed appropriate by their physician (without having to use other medications not considered the best option[s] for them) may also be compromised in achieving desired health outcomes in a timely manner. There is clearly a need for additional research on the impact of reimbursement policies on patient outcomes. ${ }^{21}$

Medication access (ie, affordability, availability, and access in a timely manner) is but one of the factors contributing to this challenge. As articulated by the "clinical inertia" ${ }^{15-19}$ literature cited above, the time lost in getting to a point of disease control is an important issue, and we need to examine whether current medication access policies that force progressive failure on one agent before adding another is in the best interests of PWD. It is important that we ensure that health system barriers, such as cost and limited options, are not undermining the care of PWD and thus contributing to the suboptimal outcomes that have been documented for the Canadian population. 


\section{Medication access from a patient perspective: what are PWD saying they need?}

No one understands the complexity of diabetes and its management better than people who live with diabetes (PWD), their family and caregivers (FMs), and their health care providers (HCPs). The perspectives and experiences of over 8,500 PWD, 2,000 FMs, and 4,700 HCPs were captured in the second Diabetes Attitudes, Wishes, and Needs (DAWN2) study involving 17 countries and multiple national and international partners. ${ }^{22-25}$ The study applied an adapted patientcentered care (PCC) model,${ }^{26}$ emphasizing the needs of PWD in the context of availability and access to the appropriate or "right" treatment interventions and services, implemented in the "right way" at the "right time" through the "right" chronic care team-based approaches, and supported by the "right" programs including self-management education and psychosocial supports. The intent of the study was to ensure that the voices of PWD, FMs, and HCPs would be heard at national and global levels, and that the study findings and resulting PCC action platform would identify the needs and barriers to be addressed and drive innovative approaches to improving diabetes therapies and their access. ${ }^{27}$

Access to diabetes medications and supplies is considered an important factor in the success of PCC and diabetes management and, thus, one of the key areas explored in DAWN2 along with other diabetes surveys and studies carried out by Statistics Canada and the CDA. ${ }^{28,29}$ In DAWN2, 90\% of PWD indicated that they understood that if they managed their diabetes carefully, they could avoid complications. Access to and availability of medications and supplies are also understood to be key factors influencing diabetes management and outcomes. However, depending on where PWD live (ie, which country and [in Canada] which province), they may not have the same access to medications (11\% global PWD and 5\% Canadian PWD) and supplies (11\% and 10\%, respectively), and may have worse drug or supply coverage.

As noted in the "Scope of the Canadian diabetes challenge" section, many Canadian PWD do not have insurance or coverage to pay for their prescription medications and/ or blood glucose monitoring equipment and supplies. Outof-pocket costs are a burden for these PWD, especially for those with lower or fixed incomes. Additional burdens include having to take more than one medication to treat secondary complications or other issues. According to DAWN2, about one-fifth of those with T2DM globally and one-quarter of Canadian PWD indicated that they had difficulty paying out of pocket for their diabetes medication. ${ }^{30}$
The CDA 2015 survey showed similar results and also found that the inability to pay for medications negatively impacted the ability of these individuals to adhere to their prescribed treatment plan.

HCPs in DAWN2 acknowledged the treatment access difficulties that PWD experience, and over $50 \%$ in both Canada and globally felt there was a need for improvements to access to assist PWD. On the one hand, more global (33\%) than Canadian (23\%) HCPs were concerned about the access to basic diabetes medications, while $45 \%$ global and $27 \%$ Canadian HCPs were concerned about the challenge of accessing blood glucose testing supplies and devices. On the other hand, more Canadian than global HCPs $(61 \%$ and $52 \%$, respectively) felt that affordability of diabetes medications needed to improve.

FMs, on the other hand, were worried more about hypoglycemic events and the psychosocial aspects related to living with diabetes and self-management. FMs (38\% global FMs and 28\% Canadian FMs), like PWD (39\% global and Canadian) and HCPs (63\% global and 31\% Canadian), felt that active self-management for PWD needed to improve, including taking medications as indicated. It was also noted that psychosocial factors affect how PWD make decisions about paying for or taking their medications every day as prescribed. The DAWN2 study participants identified a number of psychosocial factors which could impact diabetes self-management, including stress, depression, worry about hypoglycemic events, and the general impact of diabetes on physical health, emotional well-being, work, relationships, leisure activities, and finances. ${ }^{31}$ Diabetes education was viewed as being beneficial, particularly for appropriately supporting and managing the psychosocial aspects and diabetes management. Consideration of all of these factors is essential in PCC to ensure that PWD are provided what they need in the way of access to medications, care and supports, in order for them to self-manage their diabetes effectively and prevent complications.

These results are consistent with research by Statistics Canada and the CDA. The 2011 Statistics Canada survey ${ }^{28}$ confirmed Canadians' awareness of the serious risk diabetes presents for individuals and society, and the eminent threat it presents to personal and family health, as well as to the health care system and the Canadian economy.

\section{The ongoing evolution of data in diabetes treatment}

With the increasing global burden of diabetes, there has been a significant increase in the body of literature related 
to diabetes medications. In addition to the wealth of data available for new products, there has also been an increase in publication of outcomes data for older products that help to address questions such as those raised by Canadian payers and HTA bodies. Thanks to improvements in observational research methods, an increased focus on assessing the realworld impacts of treatments is generating important data to answer key questions that put the value of all products into perspective. (Note: The following data review does not include a summary of the literature on the cost-effectiveness of individual diabetes agents, since the majority of these analyses do not take the perspective of the Canadian payer and/or health care system. The focus here is on clinical data, given that the tailoring of diabetes treatments is a function of the relationship between a patient's clinical characteristics and the clinical data available.)

Such data help clinicians to more accurately select treatments based on the clinical profile of the individual PWD. While there are limitations to the use of observational data, the consistency in the trends seen in studies over the years cannot be ignored. From a policy perspective, understanding the real-world impacts of medications provides powerful information about the "value" that payers may or may not be achieving from their formulary access policies.

\section{Effect on blood glucose control}

A recent review confirmed the value of metformin as a firstline therapy for T2DM..$^{32}$ The authors undertook a systematic review and meta-analysis to evaluate the comparative effectiveness and safety of monotherapy (thiazolidinediones, metformin, SUs, DPP-4s, SGLT2s, GLP-1s) and selected metformin-based combinations. They concluded that the evidence supports metformin as first-line therapy for T2DM, given its relative safety and beneficial effects on $\mathrm{HbAlc}$, weight, and CV mortality (compared with SUs). They noted that the results for add-on therapies to metformin were similar to those for monotherapies.

Similar to the 2016 review above, there is a significant body of literature demonstrating a high degree of consistency for most second-line (add-on to metformin) and third-line (add-on to metformin + second-line therapy) treatments in terms of their impact on $\mathrm{HbA} 1 \mathrm{c}$ lowering and control. ${ }^{33-35}$ The advantages of newer agents tend to be related to improved side effect profiles (eg, reduced risk of hypoglycemia, infection) or other more subtle attributes that may be beneficial, given the high frequency of comorbid conditions in the PWD (eg, blood pressure lowering, weight loss).

\section{Understanding outcomes and risks}

There have been key advances in science over the years in the availability of data sources (ie, population database analyses) and new analytic methods (ie, indirect treatment comparisons) - to supplement our traditional approaches to evidence generation and analysis. These advances have contributed to a better understanding of real-world outcomes as well as comparative assessments for diabetes products and other interventions. This emerging information is very useful to clinicians and payers, and also speaks of the significant complexity of diabetes care.

From an overall diabetes outcomes perspective, initiatives are underway to generate new data and gain a greater understanding of the issues that impact successful diabetes care and management. For instance, the US Patient-Centered Outcomes Research Institute is funding studies to look at a wide range of issues related to diabetes care and outcomes (eg, patient preferences, models of care, adherence, comparative assessment of treatments). ${ }^{36}$

More specific to drug therapies, UK researchers recently published a primary care-based open cohort study that looked at various T2DM treatments and their respective risks for a wide range of adverse outcomes associated with diabetes and/or its treatment. ${ }^{37}$ Of note, compared with metformin monotherapy, triple therapy with metformin, SUs, and either gliptins or glitazones was associated with an increased risk of hypoglycemia, which was similar to the risk associated with the dual therapy with metformin and SUs.

Several studies have helped to more specifically describe and quantify the relative risk of CV events with SUs as well as other agents using real-world data. For instance, analysis of outcomes data from the UK Clinical Practice Research Datalink demonstrated a reduction in all-cause mortality for individuals treated with metformin combined with a DPP-4 versus metformin plus an SU, and a similar trend for major adverse cardiac events (MACE) ${ }^{38} \mathrm{~A}$ similar study conducted in Taiwan showed that DPP-4s were associated with lower risks for all-cause death, MACE, ischemic stroke, and hypoglycemia compared to SUs, when used as add-ons to metformin therapy. ${ }^{39}$

There has also been an increased emphasis on reassessment of the data available for traditional diabetes therapies. The availability of a wider range of diabetes treatment modalities that deliver a broader range of outcomes has started to change the benchmark for outcome expectations from diabetes treatments. These reevaluations are critical to ensuring that our attempts to make diabetes care more patient- 
centered are based on a renewed standard that reflects the factors that are important to PWD as well as payers.

For instance, some European authors recently reviewed the evidence to assess and quantify the key factors influencing mortality in T2DM, as part of an effort to provide recommendations for targets and treatment in the European region. ${ }^{40}$ A broad scope of parameters were evaluated, including age of diabetes onset, T2DM medication, compliance, HbA1c levels, concomitant diseases, gender, body mass index, smoking, alcohol consumption, socioeconomic status, level of education, and geography. The paper articulated a range of conclusions related to lifespan, with a particular emphasis on individualized treatment. On the issue of medications, the authors concluded that the risk for mortality is highest in PWD who are treated with an SU.

CADTH reviewed the effects of first- versus secondgeneration SUs in elderly individuals, with a focus on adverse outcomes. ${ }^{41}$ Limited evidence from four nonrandomized studies suggested an increased risk of progression toward adverse renal endpoints with the use of glimepiride versus gliclazide, while the results regarding the risk of progression to $\mathrm{CV}$ endpoints were conflicting. Researchers at the University of Alberta have identified mortality risk differences among various SUs, helping to refine our understanding of the known elevated risk of $\mathrm{CV}$ events with these agents compared with other antidiabetic drugs. ${ }^{42}$

In terms of newer agents, questions have been raised about the risk for hospitalized heart failure associated with DPP-4s. A recent population-based, retrospective, new-user cohort study examined the associations of heart failure with saxagliptin and sitagliptin. ${ }^{43} \mathrm{~A}$ higher risk for heart failure was not observed in users of saxagliptin or sitagliptin, compared with the other antihyperglycemic agents evaluated. However, a systematic review and metaanalysis undertaken to examine the association between DPP-4s and the risk of heart failure or hospital admission for heart failure in people with T2DM showed different results. ${ }^{44}$ The relative effect of DPP-4s on the risk of heart failure was found to be uncertain due to the relatively short follow-up and the low quality of evidence. Both randomized controlled trials and observational studies were assessed and were found to suggest that DPP-4s may increase the risk of hospital admission for heart failure in those with existing $\mathrm{CV}$ diseases or multiple risk factors for vascular diseases, compared with no use.

A recent systematic review and meta-analysis intended to quantify the risk of hypoglycemia associated with the concomitant use of DPP-4s and SUs found that adding a gliptin to an
SU led to an excess risk for hypoglycemia of $>50 \% .{ }^{45}$ While DPP-4s themselves carry a lower risk of hypoglycemia, when combined with SUs (and their well-known association with hypoglycemia), these findings reinforce the need for awareness of the side effect profiles and risks in combination treatment.

While representing only a limited selection of research related to outcomes and risks, studies such as these help to refine our understanding of the important factors and considerations when making choices about medications in a manner that is tailored to the needs of the individual PWD.

\section{Large outcome studies}

In recent years, there has been a growing interest in assessing the $\mathrm{CV}$ outcomes and mortality impacts of newer diabetes treatments, in studies involving large numbers of PWD over extended periods of time. (These are the direct results of new regulatory requirements stemming from evidence for rosiglitazone in the mid-2000s. ${ }^{46}$ ) Improving long-term CV (ie, macrovascular) outcomes and, more recently, avoiding $\mathrm{CV}$ risk through effective diabetes management have long been the "Holy Grail" in demonstrating the value of diabetes medications, particularly from a payer's/HTA's point of view. Whether these large studies have been able to answer this question is up for debate, as differences in patient populations (ie, degree of underlying CV risk), outcome measures, outcome reporting methods, and study design make it difficult to compare the results. ${ }^{47}$

A series of trials involving the DPP-4s have looked at $\mathrm{CV}$ outcomes, with mixed results due to differences in design (eg, management of the control arm), populations, and outcome measures. The first of these was the saxagliptin/ SAVOR-TIMI 53 study, ${ }^{48}$ which showed no impact of saxagliptin on the rate of ischemic events, but a higher likelihood of hospitalization for heart failure than in those on placebo. The alogliptin/EXAMINE study ${ }^{49}$ and the sitagliptin/TECOS study $^{50}$ are more recent trials that looked at heart failure and mortality outcomes. In T2DM patients with recent acute coronary syndrome, EXAMINE showed that the rates of major adverse $\mathrm{CV}$ events (composite primary endpoint: death from $\mathrm{CV}$ causes, nonfatal myocardial infarction, or nonfatal stroke) were not increased with alogliptin versus placebo. TECOS showed that adding sitagliptin to usual care did not appear to increase the risk of MACE, hospitalization for heart failure, or other adverse events in PWD and established CV disease, compared with placebo.

Recently, studies of other diabetes medication classes have presented results that go beyond the mostly "neutral" CV impact of the DPP-4s. The EMPA-REG 
OUTCOME study ${ }^{51}$ is the first of the large SGLT2 studies to report. (Other studies with results pending include the canagliflozin/CANVAS ${ }^{52}$ study and the dapagliflozin/ DECLARE-TIMI58 study. ${ }^{53}$ ) In EMPA-REG OUTCOME, ${ }^{51,54}$ empagliflozin demonstrated a lower rate of the primary composite $\mathrm{CV}$ outcome and of death from any cause versus placebo, when added to standard care in patients with high CV risk. Specifically, empagliflozin reduced the aggregate outcome of myocardial infarction, stroke, and CV death by $14 \%$ (absolute rate $10.5 \%$ vs $12.1 \%$ in the placebo group), driven mainly by a $38 \%$ reduction in $\mathrm{CV}$ death (absolute rate 3.7\% vs 5.9\%). The LEADER study is the first large GLP-1 study to report, and showed that long-term use of liraglutide in high-risk PWD resulted in a significant (13\%) reduction in MACE, driven primarily by a $22 \%$ reduction in $\mathrm{CV}$ death $(P=0.01$ and 0.007 , respectively). ${ }^{55}$

EMPA-REG OUTCOME and LEADER are the first two trials mandated by the US Food and Drug Administration to show $\mathrm{CV}$ benefit rather than just lack of harm in people with T2DM and established CV disease and/or with high CV risk. It is important for payers and HTA bodies to examine their evidence evaluation and reimbursement processes in order to ensure that clinicians are able to apply these results and tailor the care of PWD in a manner that reflects truly $\mathrm{PCC}$.

\section{Tailoring the approach to treatment}

This body of emerging information on diabetes treatments has prompted movement toward greater individualization of care. We are seeing an important shift in clinical practice guidelines, with HTA leaders such as NICE ${ }^{56}$ putting a new emphasis on the need to more effectively tailor treatment to individual needs:

1.1.1 Adopt an individualised approach to diabetes care... taking into account their personal preferences, comorbidities, risks from polypharmacy, and their ability to benefit from long-term interventions because of reduced life expectancy. ${ }^{56}$

In fact, this primary statement in the revised diabetes management guideline speaks volumes about their recognition of the need for a flexible approach, particularly in the context of multiple morbidities as seen in diabetes.

Similar to other clinical practice guidelines, the NICE algorithm ${ }^{57}$ recommends that metformin be used in the first-line treatment of diabetes when medication needs to be introduced to the care plan. Where medication intensification is required, NICE recommends adding any one of the following: DPP-4, pioglitazone, SU, SGLT2 - with the choice of drug depending on safety considerations, tolerability, the person's individual clinical circumstances, preferences and needs, available licensed indications or combinations, and cost (the latter specifically noted when choosing between drugs in the same class). The flexibility in NICE's approach in terms of providing choices for the add-on agent is clear and highly patient focused.

The American Diabetes Association Guidelines (2016) ${ }^{58}$ similarly promote a patient-centered and flexible approach to diabetes care, including the choice of pharmacological agents. Again, metformin is the first choice for monotherapy. When dual therapy is needed, there is no specific preference for one product over another; the choice is dependent on various patient- and disease-specific factors including efficacy, comorbidities, degree of hypoglycemia risk, impact on weight, side effect profile, patient preferences, and relative costs.

The 2013 CDA guidelines were recently updated on an interim basis $^{59}$ (in advance of the planned 2018 update) a) to reflect evidence from the recent EMPA-REG OUTCOME study and b) to emphasize the need for a tailored approach to treatment. The guidelines state that the choice of pharmacologic treatment agents after metformin monotherapy should be individualized based on clinical needs and product characteristics (ie, degree of hyperglycemia, risk of hypoglycemia, overweight or obesity, CV disease or multiple risk factors, and comorbidities [congestive heart failure, hepatic, renal, etc]). In addition, the guidelines now suggest that, for people with clinical CV disease in whom glycemic targets are not met on metformin monotherapy, an SGLT2 with demonstrated CV outcome benefit (ie, empagliflozin at the current time) should be added to reduce the risk of CV and all-cause mortality.

\section{How do we move toward patient centricity?}

Shifting toward enhanced PCC demands that we be more holistic and proactive in how we approach all aspects of diabetes care - including medication access - in order to optimize the impact of efforts to support PWD in managing their diabetes and related conditions. Outlined below are the potential solutions that would enable the Canadian health system to go toward the path of patient centricity.

\section{Use existing data to shift toward individualized care}

The range of medications available and the continually emerging evidence associated with each of them has brought us to the point where it is possible to more precisely tailor 
treatment for the individual PWD. Choices based on patient characteristics, drug attributes, and health outcome objectives are becoming increasingly possible with the available data. However, the rigidity of systems for accessing medications in a more individualized manner remains a stumbling block.

In addition, data from DAWN2 and similar Canadian surveys should be used to inform strategies and policies to support and drive change for diabetes prevention and management programs, and to reduce the barriers, costs, and the burden on PWD and society. DAWN2's international information on best practices, policies/strategies, and various demonstration projects can inform Canadian plans and activities. $^{60}$

\section{Integrate the preferences and experiences of PWD}

An individual's wants and needs are a key factor in the successful treatment of diabetes. In the context of diabetes medications, there are two opportunities for PWD to express their preferences and expectations. At the individual patient level, HCPs could increase their focus on eliciting the perspectives of PWD in terms of the approaches to diabetes management and the objectives related to disease control. Education and open dialogue are the key to PWD being successfully integrated into diabetes care and management decisions.

At the payer and HTA level, PWD already have the opportunity to provide input into drug funding evaluation processes, although there is room for improvement. The CDA has suggested that the scope of questions used to make decisions about drug funding needs to be broader, in order to give greater consideration to the perspectives of PWD and the clinical needs of the individual. It is proposed that patientrelevant data (ie, quality of life, the ability to tolerate the medication, the impact of side effects on the well-being and the ability to manage their diabetes, and the ability to carry out activities of daily living) should have greater weight with payers when they are making decisions about the "value" of diabetes medications.

At a broader public policy level, the findings from DAWN2 as well as other surveys should be considered in planning and implementing diabetes medication access and coverage of supplies, diabetes education, diabetes management, psychosocial supports and programs, and related strategies or policies. Studies like DAWN2, which capture the voice of PWD, FMs, and HCPs, will inform where challenges exist in the system and what is needed to develop, improve, and sustain diabetes-related PCC.

\section{Align policy approaches to help achieve the outcomes desired}

At a broad level, the CDA has recommended that affordable and timely access to medications, devices, education, and care is necessary for achieving optimal diabetes control and preventing serious complications. DAWN2 and the other surveys cited confirm that a "call to action" is required to address the needs of Canadian PWD regarding access to diabetes medications and supplies as well as other interventions (eg, education, training, and psychosocial supports for selfmanagement and prevention of secondary complications). The importance of integration of care, access, service, and education is also clear.

While many jurisdictions in Canada have developed broad plans or strategies to address diabetes (or chronic diseases in general), there is typically little to no linkage between these strategies and access to medications through federal/provincial/territorial drug programs. As noted in the "Canada's current approach to diabetes medication management: processes and impacts" section, government drug programs have been more focused on drug plan rather than health system or societal costs. Such policy "disconnects" may be contributing to the less-than-optimal access and outcomes that PWD and their providers struggle with in their disease management efforts.

More holistic provincial diabetes strategies are needed, in order to promote greater alignment and accountability between public health policies and public health programs (eg, drug programs). Reframing the role and function of drug programs so that funding decisions are based on investments in - and measuring - health outcomes would be an innovative way to ensure that government drug plan decisions contribute positively to addressing the diabetes challenge that lies ahead.

\section{Bring patient centricity to HTA reviews}

As noted in the "Canada's current approach to diabetes medication management: processes and impacts" section, there is an opportunity to broaden the perspective and factors considered in national and provincial drug review processes to make these assessments more patient centric. Decision makers are currently focused on achieving greater certainty in clinical outcomes and cost-effectiveness through clarification of longer-term effects and more attractive pricing for the new diabetes products. Thus, payers will continue to look for evidence about long-term diabetes complications and comparative effectiveness and safety in their clinical assessments, even though such information might be unavailable at the time of decision making. 
Payers should similarly be looking for and giving genuine credence to robustly gathered evidence about quality of life and patient-reported outcomes that address the impacts of diabetes that are important to PWD (ie, work, social and family activities, activities of daily living, and psychological and emotional burden). There is also a need for better characterization of the value of important aspects of treatment including weight gain and hypoglycemia. Consideration of these additional attributes would provide a more holistic and patient-centered assessment of medications that would be more aligned with an individualized approach to diabetes care.

\section{Measure what is being achieved}

DAWN2 was the first diabetes study to create a global benchmarking platform for monitoring and sharing the best practices in diabetes-related PCC and diabetes management. As such, it has the potential to be a framework for the ongoing monitoring of progress, evaluation, and measurement of strategies, activities, policy initiatives, and outcomes for PWD, the health care system, and our broader society within Canada and for comparison with other countries. To address policy needs, this needs to include research to measure both the upfront investment as well as the short- and long-term cost avoidance associated with enabling access that allows prescribers to tailor their approach to treatment of individual PWD.

\section{A "call to action"}

It is clear that Canada is "behind the eight ball" when it comes to diabetes care. The time for concrete action is now - there is much work to be done on many fronts, requiring a long-term and comprehensive approach that focuses on PCC, rather than focusing on "quick wins" to meet political objectives.

The good news is that we increasingly have the data that give us direction on how PWD will benefit from specific interventions and we have many of the tools needed to move forward. As a health system, however, we need to get better at using those tools and implementing the best practices that will help PWD and providers improve diabetes outcomes.

In the case of medications, it is clear that there are several specific actions that can be taken, which are discussed below.

\section{Realign HTA/reimbursement processes and policies with health policy priorities}

- We need to address the disconnect between the impact of diabetes (on people and the health system) and the lack of flexibility within our reimbursement systems, in order to enable timely and tailored treatment that helps us achieve the desired outcomes of drug treatment in PWD.

\section{Individualized care based on current evidence}

- The constantly changing data available in this field means that policies and processes need to be nimble in order to achieve the level of individualized care needed to achieve optimal outcomes.

- Clinical practice guidelines are frequently updated when important new data come to light. There is an opportunity to more closely align the reimbursement policy to these guidelines, in order to enable individualized care.

\section{Improve equity of medication access}

- Despite the existence of national clinical practice guidelines and national HTA processes, there is no one standard of diabetes care in Canada.

- Creating such a national standard for diabetes medication access could be the first step in standardizing other important aspects of diabetes care and, thus, moving our population to improved diabetes outcomes.

\section{Move forward together}

- Collaboration across health, social, and technology sectors through roundtable discussions has the potential to move patient-centered diabetes care forward, with innovative medicines and treatment for diabetes, best practices, sustainable health care, diabetes selfmanagement, and diabetes strategies/policies at the national and provincial levels being the priority topics for discussion.

Everyone in the "diabetes ecosystem" needs to step forward and start to take action and advocate for the changes needed to achieve the outcomes that PWD deserve.

\section{Acknowledgments}

The authors would like to thank the contributions of their fellow symposium panelists to the issues raised in this paper, as well as AstraZeneca Canada Inc.'s support of the symposium entitled "Is Canada a global outlier in the treatment and public reimbursement of medications for patients with type 2 diabetes?" at the IDF World Diabetes Congress 2015 in Vancouver on November 30, 2015. 
- Wade M Aubry, MD (Associate Clinical Professor of Medicine, Philip R. Lee Institute for Health Policy Studies, University of California; Former Medical Director, Blue Shield of California and Blue Cross Blue Shield Association, San Francisco, CA, USA)

- Lori Berard, RN, CDE (Nurse Manager, Winnipeg Regional Health Authority; Health Science Centre Winnipeg Diabetes Research Group; Winnipeg, MB, Canada).

- Stewart B Harris, CM, MD, MPH, FCFP, FACPM (Professor, CDA Chair in Diabetes Management; Ian McWhinney Chair of Family Medicine Studies, Centre for Studies in Family Medicine, Western Center For Public Health and Family Medicine, Western University; London, ON, Canada).

- Joanne Jung, BSc (Pharm), ACPR (Director, Pharmacy Services, Pacific Blue Cross; Clinical Pharmacist, Lower Mainland Pharmacy Services, Fraser Health Authority; Vancouver, BC, Canada).

\section{Disclosure}

Dr Judith L Glennie has served on advisory boards and consulting projects of AstraZeneca. Dr Katharina Kovacs Burns has served as a member of the global DAWN2 study team and the International Publications Committee sponsored and supported by Novo Nordisk. The author has received compensation for travel expenses related to meetings of the DAWN2 committees only, but no other payment. Dr Kovacs Burns is a person with diabetes. Dr Paul Oh has served on advisory boards of Amgen, AstraZeneca, Janssen, Merck, Pfizer, Roche, and Sanofi. The authors report no other conflicts of interest in this work.

\section{References}

1. Canadian Institute for Health Information (CIHI). CIHI International Comparisons: A Focus on Diabetes; 2015). Available from: https:// secure.cihi.ca/free_products/oecd-diabetes-report-2015_en.pdf. Accessed May 19, 2016.

2. Canadian Diabetes Association (CDA). 2015 Report on Diabetes Driving Change. Available from: https://www.diabetes.ca/publicationsnewsletters/advocacy-reports/2015-report-on-diabetes-driving-change. Accessed May 19, 2016.

3. Canadian Diabetes Association (CDA). Diabetes Charter for Canada. Ottawa: CDA; 2014. Available from: www.mydiabetescharter.ca. Accessed July 5, 2016.

4. Canadian Institute for Health Information (CIHI). Prescribed drug spending in Canada, 2013: a focus on public drug programs; 2015. Available from: https://secure.cihi.ca/estore/productFamily.htm?local $\mathrm{e}=\mathrm{en} \& \mathrm{pf}=\mathrm{PFC} 2896 \&$ lang=en. Accessed July 5, 2016.

5. Innovative Medicines Canada. 2016 annual report: access to new medicines in public drug plans - Canada and Comparable Countries; 2016. Available from: http://innovativemedicines.ca/canadians-facingdelayed-access-to-new-innovative-medicines-report. Accessed July 1, 2016.

6. Ontario public drug programs - how drugs are approved. Available from: http://www.health.gov.on.ca/en/pro/programs/drugs/how_drugs_ approv/how_drugs_approv.aspx. Accessed July 6, 2016.
7. Canadian Agency for Drugs and Technologies in Health (CADTH) Common Drug Review (CDR). Available from: https://www.cadth.ca/ about-cadth/what-we-do/products-services/cdr. Accessed July 5, 2016.

8. The pan-Canadian Pharmaceutical Alliance. Available from: http:// canadaspremiers.ca/en/initiatives/358-pan-canadian-pharmaceuticalalliance. Accessed July 5, 2016.

9. CADTH Optimal Use Report: Third-Line Pharmacotherapy for Type 2 Diabetes - Update; Volume 3, Issue 1B; 2013. Available from: https://www.cadth.ca/sites/default/files/pdf/OP0512_Diabetes\%20 Update_Third-line_e.pdf. Accessed July 6, 2016.

10. CADTH Common Drug Review (CDR) reports. Available from: https:// www.cadth.ca/about-cadth/what-we-do/products-services/cdr/reports. Accessed July 19, 2016.

11. CADTH Canadian Drug Expert Committee (CDEC) Final Recommendation: Dulaglutide; 2016. Available from: https://www.cadth.ca/sites/ default/files/cdr/complete/SR0462_complete_Trulicity_June_21_16_e. pdf. Accessed July 19, 2016.

12. Canadian Diabetes Association. Formulary listings for diabetes medications in Canada; 2016. Available from: http://www.diabetes. ca/publications-newsletters/advocacy-reports/provincial-territorialformulary-chart. Accessed July 1, 2016.

13. Canadian Diabetes Association 2013 clinical practice guidelines for the prevention and management of diabetes in Canada. Available from: http://guidelines.diabetes.ca/?_ga=1.159114236.730924813.14636693 50. Accessed May 19, 2016.

14. Leiter LA, Berard L, Bowering CK, et al. Type 2 diabetes mellitus management in Canada: is it improving? Can J Diabetes. 2013;37(2):82-89.

15. Khunti K, Wolden ML, Thorsted BL, Andersen M, Davies MJ. Clinical inertia in people with type 2 diabetes. Diabetes Care. 2013;36(11):3411-3417.

16. Mata-Cases M, Benito-Badorrey B, Roura-Olmeda P, et al. Clinical inertia in the treatment of hyperglycemia in type 2 diabetes patients in primary care. Curr Med Res Opin. 2013;29(11):1495-1502.

17. Rajpathak, Rajgopalan S, Engel SS. Impact of time to treatment intensification on glycemic goal attainment among patients with type 2 diabetes failing metformin monotherapy. J Diabetes Complications. 2014;28(6):831-835.

18. Strain WD, Blüher M, Paldánius P. Clinical inertia in individualising care for diabetes: is there time to do more in type 2 diabetes? Diabetes Ther. 2014;5(2):347-354.

19. Reach G. Clinical inertia, uncertainty and individualized guidelines. Diabetes Metab. 2014;40(4):241-245.

20. Després F, Forget A, Kettani F-Z, Blais L. Impact of patient reimbursement timing and patient out-of-pocket expenses on medication adherence in patients covered by private drug insurance plans. J Manag Care Spec Pharm. 2016;22(5):539-547.

21. Gomes T, Martins D, Tadrous M, et al. Self-monitoring of blood glucose levels: evaluating the impact of a policy of quantity limits on test-strip use and costs. Can J Diabetes. Epub 2016 May 17.

22. Peyrot M, Burns KK, Davies M, et al. Diabetes attitudes wishes and needs 2 (DAWN2): a multinational, multi-stakeholder study or psychosocial issues in diabetes and person-centred diabetes care. Diabetes Res Clin Pract. 2013;99(2):174-184.

23. Nicolucci A, Kovacs Burns K, Hermanns N, et al. Diabetes Attitudes, Wishes and Needs second study (DAWN2 ${ }^{\mathrm{TM}}$ ): cross-national benchmarking of diabetes-related psychosocial outcomes for people with diabetes. Diabet Med. 2013;30(7):767-777.

24. Holt RI, Nicolucci A, Kovacs Burns K, et al. Diabetes attitudes, wishes and needs second study (DAWN2 ${ }^{\mathrm{TM}}$ ): cross-national comparisons on barriers and resources for optimal care - healthcare professional perspective. Diabet Med. 2013;30(7):789-798.

25. Kovacs Burns K, Nicolucci A, Holt RI, et al. Diabetes attitudes, wishes and needs second study (DAWN2 ${ }^{\mathrm{TM}}$ ): cross-national benchmarking indicators for family members living with people with diabetes. Diabet Med. 2013;30(7):778-788.

26. Peyrot M, on behalf of DAWN2 Study Group. Multi-stakeholder study and strong action platform to promote person-centred diabetes care. Diabetes Voice. 2013;58(Special Issue 2):13-16. Available from: http://www.idf.org/ diabetesvoice/issues/volume-58-special-issue-2. Accessed May 23, 2016. 
27. Skovlund $\mathrm{S}$ and Peyrot M. The Diabetes Attitudes, Wishes, and Needs (DAWN) program: a new approach to improving outcomes of diabetes care. Diabetes Spect. 2005;18(3): 136-142. Available from: http:// dx.doi.org/10.2337/diaspect.18.3.136. Available May 23, 2016.

28. Statistics Canada. Survey on Living with Chronic Diseases in Canada (SLCDC) 2011 [Internet]. Ottawa, ON: Statistics Canada; 2011. Available from http://www23.statcan.gc.ca/imdb/p2SV.pl?Function=getSurv ey $\&$ SurvId $=84769 \&$ InstaId $=84771 \&$ SDDS $=5160$. Accessed October $25,2015$.

29. Canadian Diabetes Association (CDA). Report on diabetes: driving change; 2015. Available from: http://www.diabetes.ca/publicationsnewsletters/advocacy-reports/2015-report-on-diabetes-driving-change. Accessed July 1, 2016.

30. Kovacs Burns K, Vallis M, Ross S, Hollahan D, Hahn J. Diabetes Attitudes, Wishes and Needs Second Study (DAWN2 ${ }^{\mathrm{TM}}$ ): psychosocial experiences of Canadian family members living with people with diabetes. Diabetes Res Treat Open Access. 2015;2(2).

31. Vallis M, Kovacs Burns K, Hollahan D, Ross S, Hahn J. Diabetes Attitudes, Wishes and Needs Second Study (DAWN2 ${ }^{\mathrm{TM}}$ ): understanding diabetes-related psychosocial outcomes for Canadians with diabetes. Can J Diabetes. 2016;40(3):234-241.

32. Maruthur NM, Tseng E, Hutfless S, et al. Diabetes medications as monotherapy or metformin-based combination therapy for type 2 diabetes: a systematic review and meta-analysis. Ann Intern Med. 2016;164(11): $740-751$.

33. Goring S, Hawkins N, Wygant G, Roudaut M, Townsend R, Wood I, Barnett AH. Dapagliflozin compared with other oral anti-diabetes treatments when added to metformin monotherapy: a systematic review and network meta-analysis. Diabetes Obes Metab. 2014;16(5):433-442.

34. Tricco AC, Antony J, Khan PA, et al. Safety and effectiveness of dipeptidyl peptidase-4 inhibitors versus intermediate-acting insulin or placebo for patients with type 2 diabetes failing two oral antihyperglycaemic agents: a systematic review and network meta-analysis. BMJ Open. 2014;4:e05752.

35. Palmer SC, Mavridis D, Nicolucci A, et al. Comparison of clinical outcomes and adverse events associated with glucose-lowering drugs in patients with type 2 diabetes: a meta-analysis. JAMA. 2016;316(3): 313-324.

36. Patient-Centered Outcomes Research Institute (PCORI). Some PCORI-funded research projects focused on diabetes. Available from: www.pcori.org/research-results/some-pcori-funded-research-projectsfocused-diabetes. Accessed July 7, 2016.

37. Hippisley-Cox J, Coupland C. Diabetes treatments and risk of amputation, blindness, severe kidney failure, hyperglycaemia, and hypoglycaemia: open cohort study in primary care. BMJ. 2016;352:11450. Available from: http://dx.doi.org/10.1136/bmj.i1450. Accessed May 23, 2016.

38. Morgan CL, Mukherjee J, Jenkins-Jones S, Holden SE, Currie CJ. Combination therapy with metformin plus sulphonylureas versus metformin plus DPP-4 inhibitors: association with major adverse cardiovascular events and all-cause mortality. Diabetes Obes Metab. 2014;16(10):977-983.

39. Ou SM, Shih CJ, Chao PW, et al. Effects on clinical outcomes of adding dipeptidyl peptidase-4 inhibitors versus sulfonylureas to metformin therapy in patients with type 2 diabetes mellitus. Ann Intern Med. 2015;163(9):663-672.

40. Engelmann J, Manuwald U, Rubach C, Kugler J, Birkenfeld AL, Hanefeld M, Rothe U. Determinants of mortality in patients with type 2 diabetes: a review. Rev Endocr Metab Disord. 2016;17(1):129-137.

41. CADTH. CADTH Rapid Response Report. Glyburide, Gliclazide or Glimepiride for Elderly Patients with Type 2 Diabetes: A Review of the Clinical Effectiveness and Safety - An Update; 2015. Available from: https://www.cadth.ca/sites/default/files/pdf/htis/aug-2015/RC0693\%20 Glyburide\%20Update\%20Final.pdf. Accessed July 20, 2016.

42. Simpson SH, Lee J, Choi S, Vandermeer B, Abdelmoneim AS, Featherstone TR. Mortality risk among sulfonylureas: a systematic review and network meta-analysis. Lancet Diabetes Endocrinol. 2015;3(1):43-51.
43. Toh A, Hampp C, Reichman ME, et al. Risk for hospitalized heart failure among new users of saxagliptin, sitagliptin, and other antihyperglycemic drugs - a retrospective cohort study. Ann Intern Med. 2016;164(11):705-714.

44. Li L, Li S, Deng K, et al. Dipeptidyl peptidase-4 inhibitors and risk of heart failure in type 2 diabetes: systematic review and meta-analysis of randomised and observational studies. BMJ. 2016;352:i610. Available from: http://dx.doi.org/10.1136/bmj.i610. Accessed May 23, 2016.

45. Salvo F, Moore N, Arnaud M, et al. Addition of dipeptidyl peptidase-4 inhibitors to sulphonylureas and risk of hypoglycaemia: systematic review and meta-analysis. BMJ. 2016;353:i2231. Available from: http:// dx.doi.org/10.1136/bmj.i2231. Accessed May 23, 2016.

46. Nissen SE, Wolski K. Effect of rosiglitazone on the risk of myocardial infarction and death from cardiovascular causes. $N$ Engl J Med. 2007;356(24):2457-2471

47. Ingelfinger JR, Rosen CJ. Cardiac and renovascular complications in type 2 diabetes - is there hope? N Engl J Med. 2016;375(4):380-382.

48. Scirica BM, Bhatt DL, Braunwald E, et al. Saxagliptin and cardiovascular outcomes in patients with type 2 diabetes mellitus. N Engl J Med. 2013;369(14):1317-1326

49. White WB, Cannon CP, Heller SR, et al. Alogliptin after acute coronary syndrome in patients with type 2 diabetes. $N$ Engl J Med. 2013; 369(14):1327-1335.

50. Green JB, Bethel MA, Armstrong PW, et al. Effect of sitagliptin on cardiovascular outcomes in type 2 diabetes. NEngl J Med. 2015;373(3):232-242.

51. Zinman B, Wanner C, Lachin JM, et al. Empagliflozin, cardiovascular outcomes, and mortality in type 2 diabetes. NEngl J Med. 2015;373(22): $2117-2128$

52. Janssen Research \& Development, LLC. CANVAS - CANagliflozin cardioVascular Assessment Study (CANVAS) - a randomized, multicenter, double-blind, parallel, placebo-controlled study of the effects of JNJ-28431754 on cardiovascular outcomes in adult subjects with type 2 diabetes mellitus. Available from: https://clinicaltrials.gov/ct2/ show/NCT01032629. NLM identifier: NCT01032629. Accessed July 7, 2016.

53. AstraZeneca. Multicenter trial to evaluate the effect of dapagliflozin on the incidence of cardiovascular events (DECLARE-TIMI58) - dapagliflozin effect on cardiovascular events a multicenter, randomized, double-blind, placebo-controlled trial to evaluate the effect of dapagliflozin $10 \mathrm{mg}$ once daily on the incidence of cardiovascular death, myocardial infarction or ischemic stroke in patients with type 2 diabetes. Available from: https://clinicaltrials.gov/ct2/show/NCT01730534. NLM identifier: NCT01730534. Accessed July 7, 2016.

54. Ingelfinger J, Rosen CJ. Cardiovascular risk and sodium-glucose cotransporter 2 inhibition in type 2 diabetes. N Engl J Med. 2015;373(22): 2178-2179.

55. Marso SP, Daniels GH, Brown-Frandsen K, et al; LEADER Steering Committee on behalf of the LEADER Trial Investigators. Liraglutide and cardiovascular outcomes in type 2 diabetes. $N$ Eng $J$ Med. 2016;375(4):311-322.

56. National Institute for Health and Care Excellence (NICE). NICE guideline - type 2 diabetes in adults: management; 2015. Available from: https://www.nice.org.uk/guidance/ng28. Accessed April 17, 2016.

57. NICE. NICE Algorithm for blood glucose lowering therapy in adults with type 2 diabetes; 2015. Available from: https://www.nice.org.uk/guidance/ ng28/resources/algorithm-for-blood-glucose-lowering-therapy-in-adultswith-type-2-diabetes-2185604173. Accessed April 17, 2016.

58. American Diabetes Association. Approaches to glycemic treatment. Diabetes Care. 2016;39(Suppl 1):S52-S59.

59. Canadian Diabetes Association (CDA). Clinical practice guidelines: 2016 interim update to the guidelines - update to the pharmacologic management of type 2 diabetes; 2016. Available from: http://guidelines. diabetes.ca/2016update. Accessed July 19, 2016.

60. Thoumi A, Udayakumar K, Drobnick E, Taylor A, McClellan M. Innovations in diabetes care around the world: case studies of care transformation through accountable care reforms. Health Aff. 2015;34(9): 1489-1497. 
ClinicoEconomics and Outcomes Research is an international, peerreviewed open-access journal focusing on health technology assessment, pharmacoeconomics and outcomes research in the areas of diagnosis, medical devices, and clinical, surgical and pharmacological intervention. The economic impact of health policy and health systems organization also constitute important areas of coverage. The manuscript management system is completely online and includes a very quick and fair peer-review system, which is all easy to use. Visit http://www.dovepress.com/testimonials.php to read real quotes from published authors.

Submit your manuscript here: https://www.dovepress.com/clinicoeconomics-and-outcomes-research-journal 